\title{
Therapeutic and Transmission-Blocking Efficacy of Dihydroartemisinin / Piperaquine and Chloroquine against Plasmodium vivax Malaria, Cambodia
}

\section{Jean Popovici, Amelie Vantaux, Lyse Primault, Reingsey Samreth, Eak Por Piv, Sophalai Bin, Saorin Kim, Dysoley Lek, David Serre, Didier Menard}

We assessed the efficacy of standard 3-day courses of chloroquine and dihydroartemisinin/piperaquine against Plasmodium vivax malaria. Compared with chloroquine, dihydroartemisinin/piperaquine was faster in clearing asexual $P$. vivax parasites and blocking human-to-mosquito transmission. This drug combination was also more effective in preventing potential recurrences for $\geq 2$ months.

P lasmodium vivax is the most widespread human malaria parasite. Almost 2.5 billion persons are at risk for infection in $>90$ countries $(1,2)$. Since the 1950s-1960s, Southeast Asia has been the cradle of emergence and spread of $P$. falciparum antimalarial drug resistance, a major obstacle for malaria control. Over the past decade, control efforts in Cambodia have led to an impressive decrease in malaria burden, with a slower decrease of $P$. vivax than for P. falciparum (3).

$P$. vivax resistance to chloroquine has emerged more recently; the first cases were observed in 2009 in Rattanakiri Province in northeastern Cambodia $(17.4 \%$ treatment failures after 28 days of follow-up), which led to withdrawal of chloroquine and use of dihydroartemisinin/piperaquine (DHA/PPQ) as first-line therapy for uncomplicated $P$. vivax malaria in 2012 (4). We assessed the efficacy of standard 3 -day courses of chloroquine and DHA/PPQ for treating $P$. vivax malaria, preventing recurrences, and blocking human-to-mosquito transmission.

\section{The Study}

We conducted an open-label, randomized, control trial in June-December 2014 in Rattanakiri Province, Cambodia. Febrile patients or patients with a history of fever in the

Author affiliations: Institut Pasteur, Phnom Penh, Cambodia

(J. Popovici, A. Vantaux, L. Primault, R. Samreth, E.P. Piv, S. Bin,

S. Kim, D. Menard); National Center for Malaria Control, Phnom

Penh (D. Lek); University of Maryland School of Medicine, Baltimore,

Maryland, USA (D. Serre); Institut Pasteur, Paris, France (D. Menard)

DOI: https://doi.org/10.3201/eid2408.170768 previous $48 \mathrm{~h}$ who sought treatment in health facilities and had positive results by rapid diagnostic test (CareStart Malaria HRP2/pLDH Pf/PAN Combo; Access Bio, Inc., Somerset, NJ, USA) for non- $P$. falciparum malaria were offered participation in the study. Pregnant or lactating women and patients with signs of severe malaria, other known illnesses, or inability to provide informed consent were excluded. Patients with $P$. vivax monoinfection confirmed by PCR were eligible for the study (5).

At enrollment, after we obtained written informed consent, patients were randomized to receive supervised standard 3-day courses of DHA/PPQ (Duo-Cotecxin; Zhejiang Holley Nanhu Pharmaceutical Co., Ltd., Jiaxing, China) or chloroquine (Nivaquine; Sanofi-Aventis, Paris, France). For each participant, medical histories were obtained and clinical and biological examinations performed. We followed up with patients according to an extended World Health Organization protocol on days $1,2,3,5$, and 7 and then weekly until day 63. At each visit, we performed clinical examinations and obtained an axillary temperature and a capillary blood sample.

Malaria parasites were detected by microscopy (Giemsastained blood films) and PCR as described (5). Chloroquine resistance was ruled out for patients if no parasites were detected by microscopy on day 28 or, in case of recurrence, if the chloroquine blood concentration on the day of recurrence did not exceed $>100 \mathrm{ng} / \mathrm{mL}(6,7)$. We measured chloroquine blood concentrations by using liquid chromatography-tandem mass spectrometry for $50-\mu \mathrm{L}$ samples of whole blood.

During January-March 2016, we conducted an additional study at the same site to evaluate the infectivity of $P$. vivax from blood of symptomatic patients to Anopheles dirus mosquito vectors; we tested pretreatment and posttreatment blood samples by using membrane feeding assays without serum replacement (8). Any febrile patients seeking antimalarial treatment with similar inclusion/exclusion criteria described previously were enrolled in this study.

After we obtained written informed consent, we fed batches of $50 \mathrm{An}$. dirus mosquitoes with blood collected from these patients on 3 occasions: 1) before the first dose of DHA/PPQ or chloroquine, 2) on the same day at 9:00 PM (i.e., $2-11 \mathrm{~h}$ after treatment), and 3 ) at $24 \mathrm{~h}$ posttreatment for patients treated with chloroquine. We performed statistical analyses by using GraphPad Prism 5 (GraphPad, San Diego, 
CA, USA) and R software (9). Both studies were approved by the Cambodian National Ethic Committee (038 NECHR, 2/24/2014 and 475 NECHR, 12/28/2015).
For the drug comparison study, we enrolled 50 patients (25 in each study arm); in each arm, 5 patients were lost to follow-up during days $2-35$. A total of 40 patients (20 in each

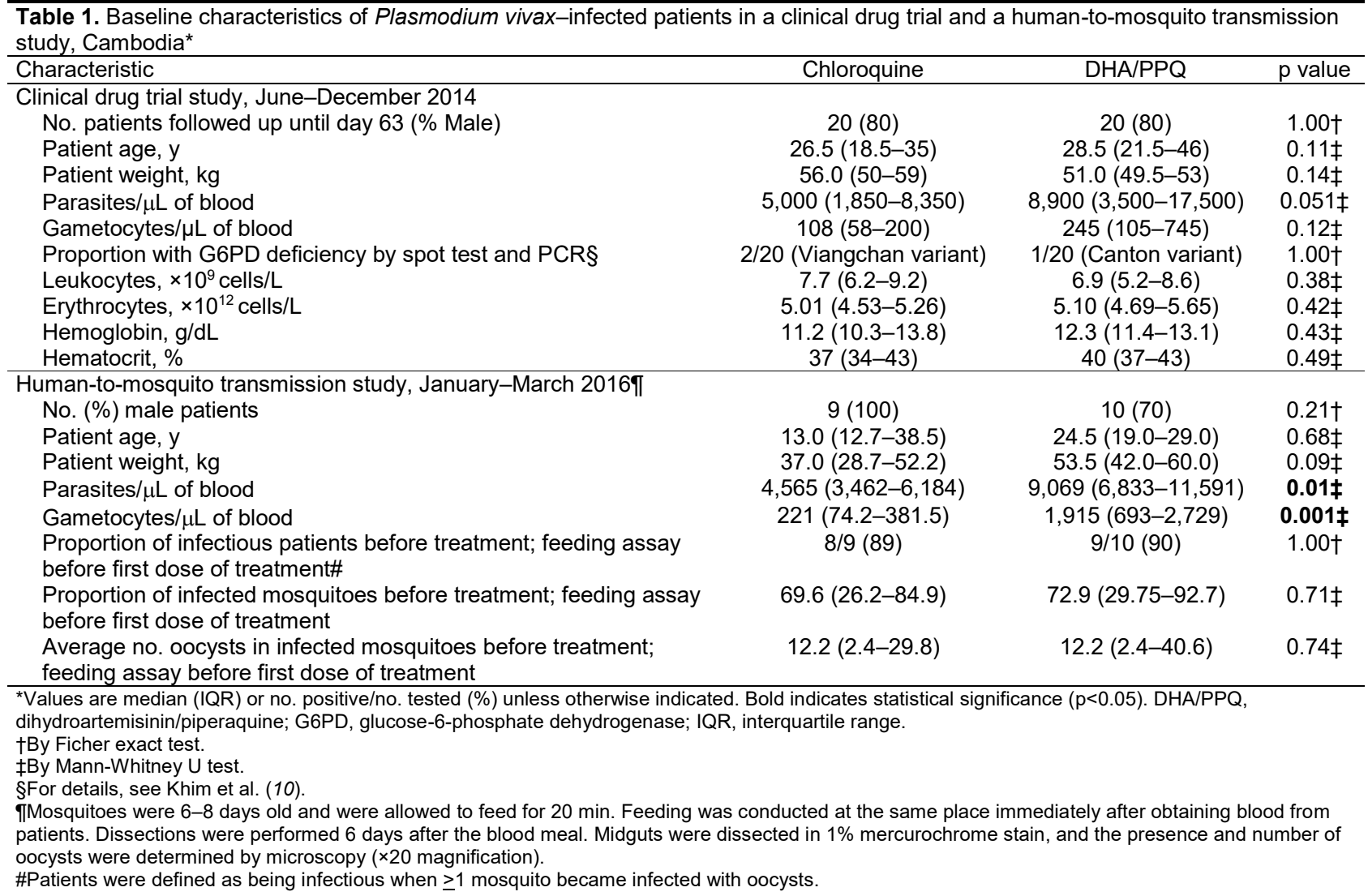

Table 2. Plasmodium vivax clearance among infected patients, time to malaria recurrence, and vector transmission results, by allocated antimalarial drug treatment, Cambodia*

\begin{tabular}{|c|c|c|c|}
\hline Characteristic & Chloroquine & DHA/PPQ & $\mathrm{p}$ value \\
\hline \multicolumn{4}{|l|}{ Clinical drug trial study, June-December 2014} \\
\hline Proportion of patients parasitemic at day 1 by microscopy & 17/20 (85) & $6 / 20(30)$ & $0.001 \dagger$ \\
\hline Parasites $/ \mu \mathrm{L}$ of blood at day 1 & $180(80-600)$ & $0(0-57)$ & $0.0002 \ddagger$ \\
\hline Parasite reduction ratio at day & $96.2(68.5-98.6)$ & $100(99.4-100)$ & $0.0002 \ddagger$ \\
\hline Proportion of patients parasitemic at day 2 by microscopy & $5 / 20(25)$ & $0 / 20(0)$ & $0.047 \dagger$ \\
\hline Parasites $/ \mu \mathrm{L}$ of blood at day 2 & $0(0-15)$ & 0 & $0.03 \ddagger$ \\
\hline Parasite reduction ratio at day 2 & $100(99.8-100)$ & 100 & $0.03 \ddagger$ \\
\hline Proportion of patients parasitemic at day 3 by microscopy & $0 / 20(0)$ & $0 / 20(0)$ & $1.00 \dagger$ \\
\hline Proportion of patients with recurrence detected by PCR & $12 / 20(60)$ & $4 / 20(20)$ & $0.02 \dagger$ \\
\hline Time to recurrence, $\mathrm{d}$ & $49(42-49)$ & $56(52.5-56)$ & $0.04 \ddagger$ \\
\hline \multicolumn{4}{|l|}{ Human-to-mosquito transmission study, January-March 2016} \\
\hline $\begin{array}{l}\text { Proportion of infectious patients after first dose of treatment; feeding assay } \\
\text { at 9:00 PM }\end{array}$ & $8 / 9(89)$ & $1 / 10(10)$ & $0.001 \dagger$ \\
\hline $\begin{array}{l}\text { Proportion of infected mosquitoes after first dose of treatment; feeding assay } \\
\text { at 9:00 PM }\end{array}$ & $60.7(23.7-78.6)$ & 0 & $0.004 \ddagger$ \\
\hline $\begin{array}{l}\text { Average no. of oocysts in infected mosquitoes after first dose of treatment; } \\
\text { feeding assay at 9:00 PM }\end{array}$ & $9.9(4.1-25.7)$ & 356.4 & $0.22 \ddagger$ \\
\hline Parasite transmissibility reduction ratio (\%) at 9:00 PM & $19(-13.8$ to 62.7$)$ & 100 & $0.003 \ddagger$ \\
\hline Proportion of infectious patient $24 \mathrm{~h}$ after first dose of chloroquine & $2 / 9(22)$ & ND & ND \\
\hline $\begin{array}{l}\text { Average no. of oocysts in infected mosquitoes after first dose of treatment for } \\
2 \text { infectious patients; feeding assay } 24 \mathrm{~h} \text { after first dose of chloroquine }\end{array}$ & 1.3 and 2.4 & ND & ND \\
\hline Proportion of infected mosquitoes $24 \mathrm{~h}$ after first dose of chloroquine & $0(0-4.4)$ & ND & ND \\
\hline
\end{tabular}
*Values are no. positive/no. tested (\%) or median (IQR) unless otherwise indicated. Bold indicates statistical significance $(p<0.05)$. DHA/PPQ, dihydroartemisinin/piperaquine; IQR, interquartile range; ND, no data.

†By Fisher exact test.

$\ddagger$ By Mann-Whitney U test. 


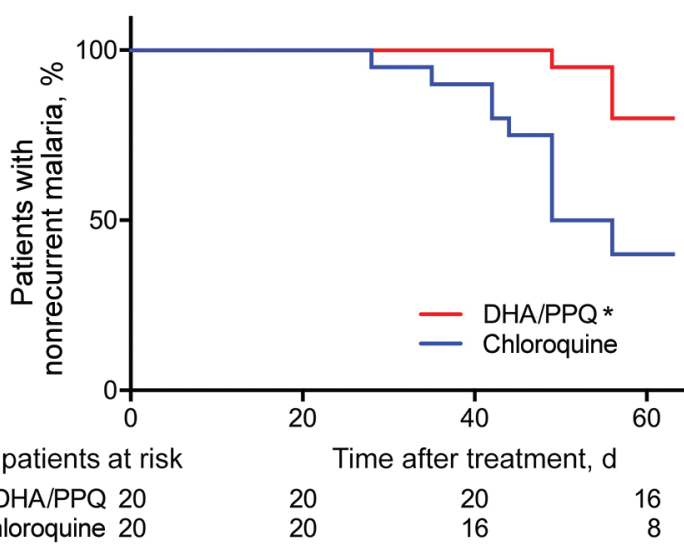

Figure 1. Cumulative proportion of patients with nonrecurrent Plasmodium vivax malaria given a 3-day course of DHA/PPQ and chloroquine detected by PCR within 63 days of follow-up, Cambodia. ${ }^{*} p<0.01$, by log-rank test during Kaplan-Meier survival analysis. DHA/PPQ, dihydroartemisinin/piperaquine.

study arm) were followed up until day 63. Baseline patient characteristics were similar for both patient groups (Table $1)$. We did not observe any adverse events or early clinical failures. The proportion of patients still parasitemic on days 1 and 2 (detected by microscopy) was lower for the DHA/ PPQ-treated group than for the chloroquine-treated group (Table 2). Medians of the parasite reduction ratio recorded on days 1 and 2 were higher for the DHA/PPQ-treated patient group (Table 2). All patients, regardless of their treatment, were microscopically parasite free at day 3 .

Within 2 months of follow up, there were fewer patients with a recurrence (detected by PCR) in the DHA/PPQtreated group than in the chloroquine-treated group (odds ratio $0.17,95 \%$ CI $0.05-0.66 ; \mathrm{p}<0.05$, by log-rank test, $\mathrm{p}<0.01$ by Kaplan-Meier survival analysis) (Table 2; Figure 1). Median time to recurrence after treatments was also delayed in patients given DHA/PPQ (56 days) compared with those patients given chloroquine (49 days) (Table 2). No recurrence occurred before day 28 in either study arm, which is suggestive of relapse or reinfection, rather than recrudescence of drug-resistant parasites $(6,7)$. In the chloroquine-treated group, $12 / 20$ patients with recurrence had a chloroquine blood concentration $<100 \mathrm{ng} / \mathrm{mL}$ on the day of recurrence (chloroquine + desethyl chloroquine: median $55.6 \mathrm{ng} / \mathrm{mL}$, interquartile range $40.0-61.7 \mathrm{ng} / \mathrm{mL}$ ); these results excluded likely chloroquine resistance $(6,7)$.

For the mosquito-to-human transmission study, we enrolled 19 patients ( 9 given chloroquine and 10 given DHA/PPQ). Baseline patient characteristics were similar in both patient groups, except for day 0 parasitemia and gametocytemia, which were higher for the DHA/PPQtreated group (Table 1). The proportion of infectious blood from $P$. vivax-infected patients and the median proportion of infected mosquitoes fed on blood collected before the

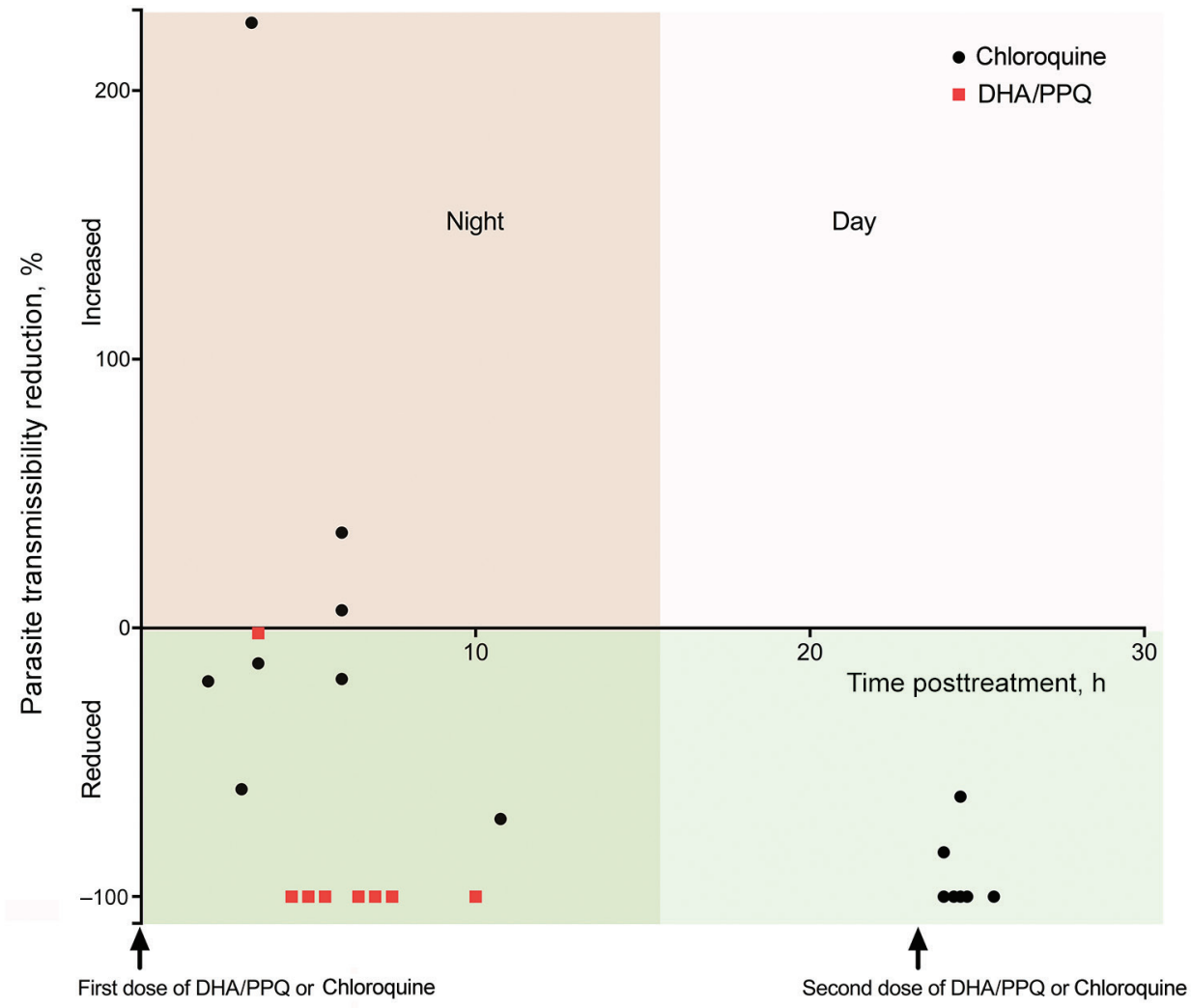

Figure 2. Transmission-blocking efficacy of allocated antimalarial drug treatment (chloroquine and DHA/PPQ) on human-to-mosquito transmission of Plasmodium vivax, January-March 2016, Cambodia. Each dot represents the parasite transmissibility reduction ratio (i.e., 100 - [proportion of infected mosquitoes fed with blood samples collected at 9:00 PM after the first dose of treatment $\times 100 /$ proportion of infected mosquitoes fed with blood samples collected at patient enrollment before the first dose of treatment]). Only data of infectious patients at enrollment are shown (17/19 patients; Table 1). For the chloroquine-treated patient group, a second mosquito blood feeding was performed 24 h after the first dose. DHA/PPQ, dihydroartemisinin/piperaquine. 
first dose of DHA/PPQ or chloroquine were similar for both patient groups (Table 1). Despite an initial higher day 0 gametocytemia for the DHA/PPQ-treated group, the proportions of infectious $P$. vivax blood collected at 9:00 PM after the first dose of DHA/PPQ or chloroquine and the median proportion of infected mosquitoes were lower for the DHA/PPQ-treated group than for the chloroquinetreated group (Table 2). Overall, DHA/PPQ acted faster than chloroquine in decreasing over time the proportion of infectious patients (generalized linear mixed model time for drug interaction, $\chi_{1}^{2}=113.1, \mathrm{p}<0.0001$ ) (Figure 2). For the group given chloroquine, $2(22 \%)$ of 9 blood samples were still infectious 24 hours after the first dose (Table 2).

\section{Conclusions}

We confirm that DHA/PPQ acts faster $(\leq 48 \mathrm{~h})$ than chloroquine $(\approx 72 \mathrm{~h})$ in eliminating sexual and asexual $P$. vivax parasites and that DHA/PPQ provides an excellent postexposure prophylaxis against potential recurrences for $\geq 2$ months (11). This benefit relies on the combination of artemisinin derivatives (DHA), which are fast-acting drugs capable of eliminating any $P$. vivax blood stages, and a long-lasting partner drug (PPQ), which has a long terminal elimination half-life and is highly effective in preventing $P$. vivax recurrence for up to 56 days. Although the number of patients enrolled was small, we demonstrated that DHA/PPQ also acts faster $(<5 \mathrm{~h})$ than chloroquine in killing $P$. vivax sexual stages and thus prevents the risk for transmission of parasites to the mosquito vector the night after uptake of the first dose. This rapid clearance of gametocytes is a major benefit of DHA/PPQ in comparison with chloroquine, given that $P$. vivax gametocytes appear early in the course of disease and must be eliminated as soon as possible to limit risk of transmission $(12,13)$.

In summary, our findings support the recommendation of DHA/PPQ as first-line treatment for P. falciparum and $P$. vivax uncomplicated malaria in regions to which these species are co-endemic. These findings apply to areas in which chloroquine is still effective and no $P$. falciparum resistance to PPQ has been observed.

\section{Acknowledgments}

We thank all patients for participating in this study.

This study was supported by the National Institute of Allergy and Infectious Diseases, National Institutes of Health (grant R01 434 A 103328) and the Institut Pasteur (grant PTR 2014-490). A.V. was supported by a Calmette and Yersin fellowship from the Institut Pasteur International Network.

\section{About the Author}

Dr. Popovici is a research scientist at the Institut Pasteur, Phnom Penh, Cambodia. His primary research interests are a better understanding of $P$. vivax malaria and providing more adapted control solutions to policy makers.

\section{References}

1. Battle KE, Gething PW, Elyazar IR, Moyes CL, Sinka ME, Howes RE, et al. The global public health significance of Plasmodium vivax. Adv Parasitol. 2012;80:1-111. http://dx.doi.org/10.1016/B978-0-12-397900-1.00001-3

2. Gething PW, Elyazar IR, Moyes CL, Smith DL, Battle KE, Guerra CA, et al. A long neglected world malaria map: Plasmodium vivax endemicity in 2010. PLoS Negl Trop Dis. 2012;6:e1814. http://dx.doi.org/10.1371/journal.pntd.0001814

3. Siv S, Roca-Feltrer A, Vinjamuri SB, Bouth DM, Lek D, Rashid MA, et al. Plasmodium vivax Malaria in Cambodia. Am J Trop Med Hyg. 2016;95(6 Suppl):97-107. http://dx.doi.org/ 10.4269/ajtmh.16-0208

4. Leang R, Barrette A, Bouth DM, Menard D, Abdur R, Duong $\mathrm{S}$, et al. Efficacy of dihydroartemisinin-piperaquine for treatment of uncomplicated Plasmodium falciparum and Plasmodium vivax in Cambodia, 2008 to 2010. Antimicrob Agents Chemother. 2013;57:818-26. http://dx.doi.org/10.1128/ AAC.00686-12

5. Canier L, Khim N, Kim S, Sluydts V, Heng S, Dourng D, et al. An innovative tool for moving malaria PCR detection of parasite reservoir into the field. Malar J. 2013;12:405. http://dx.doi.org/ 10.1186/1475-2875-12-405

6. Baird JK, Leksana B, Masbar S, Fryauff DJ, Sutanihardja MA, Suradi, et al. Diagnosis of resistance to chloroquine by Plasmodium vivax: timing of recurrence and whole blood chloroquine levels. Am J Trop Med Hyg. 1997;56:621-6. http://dx.doi.org/10.4269/ ajtmh.1997.56.621

7. World Health Organization. Methods for surveillance of antimalarial drug efficacy, 2009 [cited 2018 Mar 27]. http://apps.who.int/iris/handle/10665/44048

8. Bousema T, Dinglasan RR, Morlais I, Gouagna LC, van Warmerdam T, Awono-Ambene PH, et al. Mosquito feeding assays to determine the infectiousness of naturally infected Plasmodium falciparum gametocyte carriers. PLoS One. 2012;7:e42821. http://dx.doi.org/10.1371/journal.pone.0042821

9. R Development Core Team. R: a language and environment for statistical computing. Vienna: R Foundation for Statistical Computing; 2013.

10. Khim N, Benedet C, Kim S, Kheng S, Siv S, Leang R, et al. G6PD deficiency in Plasmodium falciparum and Plasmodium vivax malaria-infected Cambodian patients. Malar J. 2013;12:171. http://dx.doi.org/10.1186/1475-2875-12-171

11. Sinclair D, Gogtay N, Brand F, Olliaro P. Artemisinin-based combination therapy for treating uncomplicated Plasmodium vivax malaria. Cochrane Database Syst Rev. 2011; (7):CD008492.

12. Sagara I, Beavogui AH, Zongo I, Soulama I, Borghini-Fuhrer I, Fofana B, et al. Safety and efficacy of re-treatments with pyronaridine-artesunate in African patients with malaria: a substudy of the WANECAM randomised trial. Lancet Infect Dis. 2016;16:189-98. http://dx.doi.org/10.1016/S1473-3099 (15)00318-7

13. Sawa P, Shekalaghe SA, Drakeley CJ, Sutherland CJ, Mweresa CK, Baidjoe AY, et al. Malaria transmission after artemetherlumefantrine and dihydroartemisinin-piperaquine: a randomized trial. J Infect Dis. 2013;207:1637-45. http://dx.doi.org/10.1093/ infdis/jit077

Address for correspondence: Didier Menard, Malaria Genetics and Resistance Group, Institut Pasteur, 25-28 Rue du Dr. Roux, 75724 Paris CEDEX 15, France; email: dmenard@pasteur.fr 